\title{
Pirolisis convencional de neumáticos en desuso para la producción de combustibles líquidos
}

\section{Conventional disused tire pyrolysis for the production of liquid fuels}

\author{
*Duarte, S. ${ }^{1}$; Velázquez, E. ${ }^{1}$; Dullak, A. ${ }^{2}$ \\ ${ }^{1}$ Dpto. Aplicaciones Industriales, Facultad de Ciencias Químicas, Universidad \\ Nacional de Asunción. San Lorenzo, Paraguay \\ ${ }^{2}$ Facultad de Ciencias Químicas, Universidad Nacional de Asunción. San Lorenzo, \\ Paraguay
}

\section{RESUMEN}

Las características del producto líquido obtenido de la pirolisis de neumáticos en desuso, en las mejores condiciones seleccionadas a partir de un diseño factorial $2^{3}$ con puntos centrales, en el rango de 450 a $550{ }^{\circ} \mathrm{C}, 10$ a $20^{\circ} \mathrm{C} / \mathrm{min}$ y 1 a $3 \mathrm{~mm}$ de tamaño de partícula, fueron evaluadas luego de $40 \mathrm{~min}$ de pirolisis isotérmica. El modelo matemático obtenido mediante regresión, indica con un $95 \%$ de nivel de confianza, que la tasa de calentamiento y sus interacciones, no tienen un efecto estadísticamente significativo en el rendimiento del producto líquido, dentro del espacio de búsqueda del experimento. Las características del producto líquido de densidad, viscosidad, punto de inflamación, índice de cetanos, contenido de azufre y poder calorífico, indican que el mismo, posee características combustibles similares a las del diésel, pero necesita refinamiento previo en su contenido de azufre, el cual resultó ser más de 100 veces superior al máximo permitido en Paraguay, para ser aplicado a los motores diésel modernos. Adicionalmente fueron analizadas las características combustibles del residuo sólido del proceso, presentando semejanzas con el carbón mineral antracita, con un poder calorífico de 29,3 MJ/kg.

Palabras clave: pirolisis, combustibles, neumáticos en desuso, diseño factorial, modelo matemático.

\section{ABSTRACT}

Characteristics of the liquid product obtained from waste tire pyrolysis were assessed after 40 minutes of isothermal pyrolysis. The process was carried out in conditions ranging 450 to $550{ }^{\circ} \mathrm{C}, 10$ to $20^{\circ} \mathrm{C} / \mathrm{min}$ and 1 to $3 \mathrm{~mm}$ particle size, according to a $2 \wedge 3$ factorial design with center point runs. The regression model indicates that, within a confidence interval of $95 \%$, the heating rate and its interactions have no statistically significant effect in the liquid product yield, within the search space of the experiment. The liquid product's density, viscosity, flash point, cetane index, and heating value resemble those of diesel fuel. Nonetheless it needs previous refinement to reduce its sulfur content, a hundred times larger than the specified in Paraguayan standards, prior to its use in modern diesel engines. Additionally, the solid product's characteristics resemble those of anthracite, with a higher heating value of $29.3 \mathrm{MJ} / \mathrm{kg}$.

Keywords: pyrolysis, fuels, waste tires, factorial design, mathematical model.

*Autor Correspondiente: Shirley Duarte. Dpto. Aplicaciones Industriales, Facultad de Ciencias Químicas, Universidad Nacional de Asunción. San Lorenzo, Paraguay

E-mail: sduarte@qui.una.py

Fecha de recepción: setiembre 2016; Fecha de aceptación: octubre 2016. 


\section{INTRODUCCIÓN}

El agotamiento gradual de los combustibles fósiles, sumado a la creciente tendencia de consumo de energía en el mundo, crea una necesidad urgente de encontrar combustibles alternativos viables y amigables al ambiente que puedan sustituir a los derivados del petróleo (Kumaravel et al., 2016).

Por otro lado, el notable incremento en el número de vehículos a nivel mundial, las cubiertas en desuso que los mismos pueden generan, elementos ideales para servir de criaderos de insectos y la falta de mecanismos tanto técnicos como económicos para su disposición, hacen que los neumáticos en desuso sean considerados un problema de polución grave, ya que su quema contamina el aire y causa un daño excesivo para la salud humana, siendo las principales emisiones de contaminantes los hidrocarburos poliaromáticos (PAH), benceno, estireno, butadieno y sustancias de fenol (Aydın \& Ilkılıç, 2012); mientras que su entierro contamina la capa freática por varias décadas.

Se han investigado diversas formas de recuperar energía a partir de materiales de desecho, incluyendo materiales no biodegradables, residuos sólidos municipales, desechos industriales, agrícolas, así como también materiales de alta densidad energética, tales como el caucho y plásticos (Wang et al., 2010), presentando la pirólisis, una ventaja importante desde el punto de vista del reciclaje, ya que permite tratar residuos que de otra forma serían difícilmente reciclables, y obtener productos que se pueden volver a utilizar (Martínez et al., 2013).

Aunque existen varios estudios sobre los mecanismos de degradación térmica de los componentes principales de los neumáticos (Blazsó, 2006), que demuestran el efecto que tienen las condiciones de operación sobre el rendimiento del producto líquido; que altas temperaturas favorecen la formación de gases (Martínez, 2013; Miranda, 2013), que tamaños de partícula menores aumenta la volatilización en detrimento del producto sólido (Dai et al., 2001), ninguno es específico para un residuo real de neumáticos en desuso, ninguno emplea un enfoque factorial que tome en cuenta la posible interacción entre los factores, para el espacio de búsqueda empleado en este experimento.

En este trabajo, el efecto de la temperatura $(T)$, tasa de calentamiento (tc) y tamaño de partícula (TP) en el rendimiento del producto líquido, para la pirólisis convencional, fue estudiado. Inmediatamente después, un modelo matemático apropiado fue estimado. Asimismo, los rendimientos de los diferentes productos: sólidos, líquidos y gaseosos, fueron registrados. Además, el principal enfoque del trabajo fue, para una condición seleccionada, evaluar las características de los productos sólido y líquido obtenidos, para su empleo directo como materiales combustibles.

\section{MATERIALES Y MÉTODO}

Los neumáticos triturados fueron provistos por la empresa Nasser Cubiertas S.A.C.I. ubicada en Ciudad del Este, Paraguay. La materia prima fue caracterizada previamente, en los laboratorios del Instituto Nacional de Tecnología y Normalización del Paraguay (INTN), mediante análisis próximo.

Las muestras limpias fueron tamizadas y separadas en fracciones de entre 1 a $3 \mathrm{~mm}$. La pirólisis fue realizada a presión atmosférica, en un sistema reactorcondensador de construcción local. Cada muestra, con un peso inicial de $40 \pm$ $0,5 \mathrm{~g}$ fue dispuesta en un reactor de lecho fijo, tubular de $15 \mathrm{~cm}$ de longitud y $3,54 \mathrm{~cm}$ de diámetro interno, cuyos extremos fueron sellados mediante telas metálicas para soportar el lecho. Se utilizó una corriente de nitrógeno igual a 2 $\mathrm{L} \mathrm{min}^{-1}$ como gas de arrastre durante todo el proceso. 
El reactor fue calefaccionado en un horno eléctrico. A la salida del reactor, los gases y vapores fueron arrastrados a un intercambiador de tubos concéntricos de acero inoxidable, empleando agua a temperatura ambiente en contracorriente, como fluido refrigerante. A la salida del condensador, la fracción de vapores condensables a temperatura ambiente, fue colectada. Los gases no condensables, tras ser direccionados a un separador líquido-gas, por una conexión lateral al colector, fueron liberados al exterior, a través de una manguera de resina vinílica.

Se utilizó un diseño experimental $2^{3}$ con corridas en el punto central. Se llevaron a cabo dos réplicas en cada punto factorial y tres en el punto central de modo a comprobar la repetitividad en los puntos factoriales y poder estimar de forma precisa el error experimental y brindar estabilidad al diseño. Las 19 corridas fueron realizadas en orden aleatorio. El software empleado para el análisis estadístico de los datos, fue el Minitab 17®. Los factores y niveles utilizados fueron los presentados en la Tabla 1.

Tabla 1. Codificación para el diseño factorial

\begin{tabular}{lccc}
\hline & \multicolumn{3}{c}{ Niveles } \\
\hline Factor & $\mathbf{- 1}$ & $\mathbf{0}$ & $\mathbf{+ 1}$ \\
\hline Temperatura $\left[{ }^{\circ} \mathrm{C}\right]$ & 450 & 500 & 550 \\
Tamaño de partícula $[\mathrm{mm}]$ & 1 & 2 & 3 \\
Tasa de calentamiento $\left[{ }^{\circ} \mathrm{C} / \mathrm{min}\right]$ & 10 & 15 & 20 \\
\hline
\end{tabular}

La respuesta medida fue el porcentaje en peso de la muestra original que se pudo recuperar como líquidos, es decir, el rendimiento en líquidos, calculada mediante la Ec. 1.

$$
\text { Rendimiento }(L)=\frac{m_{l}}{m_{i}} \times 100
$$

donde $m_{l}$ es la masa de líquidos producida durante el proceso y $m_{i}$ es la masa inicial de la muestra.

Se realizó el análisis de varianza de los resultados y se ajustaron a una superficie de respuesta de la forma (Ec. 2):

$$
\begin{array}{r}
y=b_{0}+b_{1} x_{1}+b_{2} x_{1}+b_{3} b_{3}+b_{11} x_{1}{ }^{2}+b_{22} x_{2}{ }^{2}+b_{33} x_{3}{ }^{2}+b_{12} x_{1} x_{2}+b_{13} x_{1} x_{3}+ \\
b_{23} x_{2} x_{3} \text { Ec. } 2
\end{array}
$$

Se evaluaron las propiedades físicas y químicas de los líquidos de pirólisis en las condiciones que arrojaron mejor rendimiento. Los análisis fueron realizados en el INTN (Instituto Nacional de Tecnología, Normalización y Metrología), donde se especifican las normas empleadas para cada determinación. Los parámetros determinados fueron: densidad (ASTM D4052-11), viscosidad (ASTM D445-15), poder calorífico (ASTM D4809-13), punto de inflamación (ASTM D93-15), contenido de azufre (ASTM D4294-10), índice de cetano (CN) (ASTM D4737-10). En el residuo sólido obtenido, se evaluó sólo la fracción que arrojó el mejor rendimiento del proceso, mediante análisis próximo (ABNT-NBR $8112-86)$ y poder calorífico superior, estimado a través de la fórmula de Goutal.

$$
H H V=343 \times C+\beta \times V
$$

Ec. 3

donde HHV es el poder calorífico superior, $C$ es el contenido de carbono fijo, $\beta$ es un coeficiente obtenido como función dela materia volátil $V$. 
Los resultados obtenidos fueron comparados con la Resolución del Ministerio de Industria y Comercio del Paraguay, No 434para gasoil Tipo I y Tipo III, y la las especificaciones europeas (UNI-EN 590:2010) para combustibles de automóviles Diésel, citado por Frigo et. al, 2014.

\section{RESULTADOS Y DISCUSION}

\section{Caracterización de la materia prima}

La Tabla 1 presenta los resultados del análisis próximo de los neumáticos en desecho, obtenidos por el Instituto Nacional de Tecnología y Normalización del Paraguay (INTN).

\begin{tabular}{cccc}
\multicolumn{4}{c}{ Tabla 2. Análisis próximo de los neumáticos en desuso. } \\
\hline Humedad [\%] & Materia Volátil [\%] & Cenizas $[\%]$ & Carbono Fijo [\%] \\
\hline $\mathbf{1 , 1}$ & $\mathbf{6 6 , 0}$ & $\mathbf{6 , 2}$ & $\mathbf{2 7 , 8}$
\end{tabular}

Los valores obtenidos difieren de los encontrados para carbón, pero son similares a los valores reportados para neumáticos en la literatura (Martínez et. al, 2013;Dai et. al, 2001; González et. al, 2001; Roy et. al, 1997, Seneca et al., 1999; Ilkılıc, 2011), excepto el poder calorífico, que es sustancialmente menor a los valores típicos (27 - $40 \mathrm{MJ} \mathrm{kg} 1$ ).

\section{Estudio correlacional y análisis estadístico}

En la Tabla 3, se presentan los rendimientos para las distintas condiciones de operación en las distintas corridas.

Se puede observar el mayor rendimiento de 42,7\%, para las condiciones de $550{ }^{\circ} \mathrm{C}, 20{ }^{\circ} \mathrm{C} \mathrm{min}^{-1}$ y $1 \mathrm{~mm}$, similares a los reportados en (Aydin, 2012). El menor rendimiento de $29,6 \%$, se dio para las condiciones de $450{ }^{\circ} \mathrm{C}, 20{ }^{\circ} \mathrm{C} \mathrm{min}{ }^{-}$ ${ }^{1}$ y $3 \mathrm{~mm}$.

Tabla 3. Rendimiento del producto líquido

\begin{tabular}{|c|c|c|c|c|c|c|}
\hline \multicolumn{7}{|c|}{ Temperatura $\left[{ }^{\circ} \mathrm{C}\right]$} \\
\hline $\begin{array}{l}\text { partícula } \\
\text { [mm] }\end{array}$ & & & 500 & & & $\begin{array}{c}\text { calentamiento } \\
{\left[{ }^{\circ} \mathrm{C} \mathrm{min}^{-1}\right]}\end{array}$ \\
\hline \multirow{3}{*}{1} & 39,4 & 41,3 & & 40,2 & 41,8 & 10 \\
\hline & 39,0 & 35,9 & & 41,6 & 42,7 & 20 \\
\hline & & & 39,0 & & & 15 \\
\hline \multirow{3}{*}{2} & & & 37,1 & & & 15 \\
\hline & & & 40,6 & & & 15 \\
\hline & 30,2 & 30,0 & & 36,8 & 38,7 & 10 \\
\hline 3 & 29,9 & 29,6 & & 36,2 & 34,8 & 20 \\
\hline
\end{tabular}

Mayores temperaturas y menores tamaños de partícula parecen favorecer al rendimiento del producto líquido, pero el efecto de la tasa de calentamiento parece ser despreciable.

En la Tabla 4, se presenta el análisis de ANOVA, con un intervalo de confianza del $95 \%$, para detectar los valores no significativos. 
Tabla 4. Análisis de Varianza

\begin{tabular}{llllll}
\hline Fuente & GL & SC Ajust. & MC Ajust. & Valor F & Valor p \\
\hline Regresión & 4 & 318,399 & 79,6 & 50,30 & 0,000 \\
T & 1 & 76,606 & 76,606 & 48,41 & 0,000 \\
TP & 1 & 210,323 & 210,323 & 132,90 & 0,000 \\
T*T & 1 & 10,057 & 10,057 & 6,36 & 0,000 \\
T*TP & 1 & 21,414 & 21,414 & 13,53 & 0,024 \\
Error & 14 & 22,155 & 1,583 & & 0,020 \\
Falta de ajuste & 4 & 9,325 & 2,331 & & \\
Error puro & 10 & 12,831 & 1,283 & 1,82 & 0,02 \\
Total & 18 & 340,555 & & & \\
\hline
\end{tabular}

El análisis estadístico, confirma que la tasa de calentamiento no ejerce un efecto significativo sobre el rendimiento de obtención de líquidos, por ello, durante la construcción paso a paso del modelo fueron excluidos los términos de primer y segundo orden del mismo. De igual manera, el término de segundo orden del tamaño de partícula y los términos de interacción de la tasa de calentamiento con la temperatura y con el tamaño de partícula, fueron excluidos, debido a que estos términos no contribuyen significativamente al modelo en la región de estudio.

La regresión presentó un $\mathrm{R}^{2}$ de $94,6 \%$, quedando el modelo de la forma expresada en la Ec. 4.

$$
\text { Rendimiento }=38.91+2.20 \times T-3.63 \times(T P)-1.99 \times T^{2}+1.16 \times T \times(T P)
$$

Gráficas de los efectos principales, de interacción y de contorno, fueron generadas adicionalmente mediante el modelo matemático obtenido. Las mismas permitieron constatar el efecto cuadrático de la temperatura, el efecto lineal del tamaño de partícula, que las curvas de interacción entre la temperatura y el tamaño de partícula no son paralelas, por lo que existe interacción significativa entre estas variables y que existe un máximo para las temperaturas de entre los 500 y $550{ }^{\circ} \mathrm{C}$ donde se obtiene un mayor rendimiento para los menores tamaños de partículas.

\section{Caracterización de los productos sólidos y líquidos}

El análisis de los productos obtenidos fue realizado por el INTN y se presenta en la Tabla 5, junto con los valores de referencia de la resolución paraguaya 434/2016 del Ministerio de Industria y Comercio para el gasoil y las especificaciones europeas para diésel UNI-EN 590:2010. 
Tabla 5. Características del producto líquido obtenido

\begin{tabular}{|c|c|c|c|c|c|c|}
\hline \multirow[t]{2}{*}{ Determinación } & \multirow[t]{2}{*}{ Unidad } & \multirow[t]{2}{*}{ Valores } & \multicolumn{2}{|c|}{$\begin{array}{c}\text { Resolución No } \\
434 / 2016\end{array}$} & \multicolumn{2}{|c|}{$\begin{array}{c}\text { UNI:EN } \\
\text { 590:2010 }\end{array}$} \\
\hline & & & Mínimo & Máximo & Mínimo & Máximo \\
\hline Densidad a $15^{\circ} \mathrm{C}$ & $\mathrm{g} \mathrm{cm}^{-3}$ & 0,91 & & & 0,82 & 0,84 \\
\hline Índice de cetanos & - & 41 & 48 & & 46 & \\
\hline Punto de inflamación & ${ }^{\circ} \mathrm{C}$ & 52 & 50 & & 55 & \\
\hline $\begin{array}{l}\text { Viscosidad cinemática } \\
\text { a } 40^{\circ} \mathrm{C}\end{array}$ & $\mathrm{mm}^{2} \mathrm{~s}^{-1}$ & 6,6 & 1,9 & 5 & 2 & 4,5 \\
\hline Azufre & $\% p / p$ & $\begin{array}{l}0,59 \\
42,19\end{array}$ & & 0,005 & & 0,001 \\
\hline
\end{tabular}

Se aprecia que la densidad es superior al máximo especificado por la norma europea a semejanza de los resultados $\left(>0,9 \mathrm{~g} \mathrm{~cm}^{-3}\right.$ ) obtenidos por Frigo et al., 2014 y Ilkılıc \& Aydin, 2011. El índice de cetanos es menor a ambas especificaciones, sin embargo es superior al obtenido por Frigo et al., 2014, lo que indica que las características de la combustión del producto obtenido no serían las más adecuadas para motores diésel modernos. El punto de inflamación es mayor al especificado por la norma paraguaya pero menor al de la norma europea, sin embargo esto no implica riesgos de incendio inadmisibles en su correcto almacenamiento.

La viscosidad cinemática supera a los valores máximos de ambas especificaciones, a diferencia de los resultados $\left(<3,8 \mathrm{~mm}^{2} \mathrm{~s}^{-1}\right)$ de Frigo et al., 2014 y Ilkılıc \& Aydin, 2011. La viscosidad es un parámetro importante para los combustibles utilizados en motores diésel, ya que mayores viscosidades empeoran la calidad de aspersión durante la inyección del combustible en el cilindro. El contenido de azufre es el parámetro que más se aleja de las normas, y por mucho. Este resultado es esperado debido al alto contenido de azufre de los neumáticos, sin embargo, es mucho menor a los resultados reportados (> 0,9\% p/p), por Frigo et al., 2014 y Ilkılıc \& Aydin, 2011.

Las características del líquido obtenido son similares a las del diésel convencional. Sin embargo, su uso en motores, debe ser evaluado, pues primeramente requiere un proceso de refinamiento, especialmente un proceso de desulfuración para reducir su contenido de azufre.

Podría ser más factible el uso de los líquidos obtenidos como combustible en calderas, en reemplazo del fuel oil, que como reemplazo del diésel en vehículos.

Los resultados del análisis del producto sólido se muestra en la Tabla 6.

Tabla 6. Análisis próximo y poder calorífico del producto sólido

\begin{tabular}{|c|c|c|c|c|c|c|}
\hline \multirow[t]{2}{*}{ Determinación } & \multirow[t]{2}{*}{ Unidad } & \multirow[t]{2}{*}{ Valores } & \multicolumn{2}{|c|}{$\begin{array}{c}\text { Resolución } N^{\circ} \\
434 / 2016\end{array}$} & \multicolumn{2}{|c|}{ UNI:EN 590:2010 } \\
\hline & & & Mínimo & Máximo & Mínimo & Máximo \\
\hline Humedad & $\% p / p$ & 1,9 & & & 0,82 & 0,84 \\
\hline Cenizas & $\% \mathrm{p} / \mathrm{p}$ & 17,7 & 48 & & 46 & \\
\hline Materia Volátil & $\% \mathrm{p} / \mathrm{p}$ & 4,3 & 50 & & 55 & \\
\hline Carbono fijo & $\% p / p$ & 78 & 1,9 & 5 & 2 & 4,5 \\
\hline Poder calorífico & $M J \mathrm{~kg}^{-1}$ & 29,27 & & & & \\
\hline
\end{tabular}

El residuo sólido obtenido es un sólido carbonoso, frágil, que pulveriza fácilmente. El análisis próximo revela que posee un porcentaje muy bajo de materia volátil, similar al 2,7\% de la antracita (Donahue \& Rais, 2009). El poder calorífico se encuentra dentro del rango $\left(15,7-33,4 \mathrm{MJkg}^{-1}\right)$ citado en la 
literatura (Martínez, 2013). Su poder calorífico sugiere su empleo como combustible sólido en aplicaciones industriales. Un detalle a tener en cuenta es el alto contenido de cenizas, que es bien sabido, podrían reblandecer a altas temperaturas en los hogares y generar incrustaciones.

Se propone para trabajos futuros, evaluar la utilización del residuo sólido como adsorbente o incluso como catalizador, tras un análisis previo que confirme sus características de porosidad y área superficial.

\section{CONCLUSIONES}

(1) En las condiciones de estudio, mayores temperaturas y menores tamaños de partícula favorecieron al rendimiento del producto líquido. Sin embargo, la tasa de calentamiento no tuvo un efecto significativo sobre el rendimiento del producto liquido ni sus interacciones con las otras dos variables es estadísticamente significativa.

(2) El rendimiento del combustible liquido, mostró dependencia lineal respecto al tamaño de partícula y cuadrática respecto a la temperatura.

(3) Las características fisicoquímicas del producto líquido obtenido fueron similares a las del diésel comercial en cuanto a densidad, viscosidad, índice de cetanos, y punto de inflamación. Sin embargo, el contenido de azufre fue muy superior al permitido, debido al alto contenido inicial del mismo en los neumáticos.

(4) El sólido carbonoso obtenido como subproducto presentó características atractivas como material combustible, a partir de su análisis próximo similar al de la antracita, su elevado poder calorífico muy superior al de la madera, y un bajo contenido de humedad, lo que hace meritorio una análisis posterior para su uso en calderas.

\section{RECONOCIMIENTOS}

A los profesores Pedro Juan Torres López y Julio Cabello, por su ayuda en la instalación y configuración del controlador PID para la tasa de calentamiento. Este trabajo fue posible gracias al financiamiento del CONACYT, a través del proyecto 14-INV-385, en el marco del programa PROCIENCIA.

\section{REFERENCIAS BIBLIOGRÁFICAS}

Aydın, H., \& Ilkılıc, C. (2012). Optimization of fuel production from waste vehicle tires by pyrolysis and resembling to diesel fuel by various desulfurization methods. Fuel, $102,605-612$.

Blazsó, M. (2006). Composition of liquid fuels derived from the pyrolysis of plastics. Feedstock Recycling and Pyrolysis of Waste Plastics: Converting Waste Plastics into Diesel and Other Fuels, 315-344.

Dai, X., Yin, X., Wu, C., Zhang, W., \& Chen, Y. (2001). Pyrolysis of waste tires in a circulating fluidized-bed reactor. Energy, 26(4), 385-399.

Donahue, C. J., \& Rais, E. A. (2009). Proximate analysis of coal. J. Chem. Educ, 86(2), 222.

Frigo, S., Seggiani, M., Puccini, M., \& Vitolo, S. (2014). Liquid fuel production from waste tyre pyrolysis and its utilisation in a Diesel engine.Fuel, 116, 399-408.

González, J. F., Encinar, J. M., Canito, J. L., \& Rodríguez, J. J. (2001) Pyrolysis of automobile tyre waste. Influence of operating variables and kinetics study. Journal of Analytical and Applied Pyrolysis, 58, 667-683.

Ilkılıc, C., \& Aydın, H. (2011). Fuel production from waste vehicle tires by catalytic pyrolysis and its application in a diesel engine. Fuel Processing Technology, 92(5), 11291135.

Italian Standards Publications. UNI EN 590. 2010. Combustibles para automoción. Combustibles para motor diesel (gasóleo). Requisitos y métodos de ensayo. 
Kumaravel, S. T., Murugesan, A., \& Kumaravel, A. (2016). Tyre pyrolysis oil as an alternative fuel for diesel engines-A review. Renewable and Sustainable Energy Reviews, 60, 1678-1685.

Martínez, J. D., Puy, N., Murillo, R., García, T., Navarro, M. V., \& Mastral, A. M. (2013). Waste tyre pyrolysis-a review. Renewable and Sustainable Energy Reviews, 23, 179-213.

Ministerio de Industria y Comercio. 2016. Resolución No 434 De la Especificación de Gasoil/Diesel.

Miranda, M., Pinto, F., Gulyurtlu, I., \& Cabrita, I. (2013). Pyrolysis of rubberty rewastes: a kinetic study. Fuel, 103, 542-552.

Roy, C., Darmstadt, H., Benallal,B.,\&Amen-Chen,C.(1997).Characterization of naphtha and carbon black obtained by vacuum pyrolysis of polyisoprene rubber. Fuel processing Technology 1997; 50(1):87-103.

Senneca, O., Salatino, P., \& Chirone, R. (1999).A fast heating-rate thermogravimetric study of the pyrolysis of scrap tyres. Fuel, 78(13), 1575-1581.

Wang, H.Z., He, X.U., \& Xuan, X.J. (2010). Review of Waste Tire Reuse \& Recycling in China-current situation, problems and countermeasures. Advances in Natural Science, 2(1), 31-39. 